\title{
Luminescence Properties of ZnS:Co Nanoparticles
}

\author{
Jafarov MA*, Nasirov EF and Jahangirova SA \\ Baku State University, Baku, Azerbaijan
}

\section{Article Info}

\author{
*Corresponding author: \\ Maarif Jafarov \\ Baku State University \\ Baku, Azerbaijan \\ E-mail: maarif.jafarov@mail.ru
}

Received: October 23, 2017

Accepted: March 30, 2018

Published: April 4, 2018

Citation: Jafarov MA, Nasirov EF, Jahangirova SA. Luminescence Properties of ZnS:Co Nanoparticles. Madridge J Nanotechnol Nanosci. 2018; 3(1): 87-89.

doi: 10.18689/mjnn-1000116

Copyright: (c) 2018 The Authovr(s). This work is licensed under a Creative Commons Attribution 4.0 International License, which permits unrestricted use, distribution, and reproduction in any medium, provided the original work is properly cited.

Published by Madridge Publishers

\begin{abstract}
An aqueous solution method has been developed for synthesizing size-controlled $\mathrm{ZnS}$ :Co nanocrystals with a relatively narrow size distribution. The nanocrystal samples were characterized by UV-Vis absorption spectra and photoluminescence spectra. We prepared narrow size distribution particles under different synthesis conditions. The effect of manganese concentration on the photoluminescence properties was investigated. Luminescence intensity in different excitation wavelength correlates with different size of $\mathrm{ZnS}: \mathrm{Co}$ nanocrystals on luminescence spectra. We found that by narrowing the size distribution and doping concentration, $\mathrm{ZnS}$ :Co samples can be prepared with high luminescence intensity.
\end{abstract}

Keywords: Luminescence; Nanoparticles; Nanocrystals; Photodetector.

\section{Introduction}

Nanoscale materials researches have stimulated great interest owing to their importance in basic scientific research and potential technological applications. The synthesis of doped nanocrystals has become a major field of recent researches. ZnS:Co nanocrystals have been mainly studied due to the luminescence of the $\mathrm{Co}^{2+}$ ions inside the $\mathrm{ZnS}$ host. This is due to the fact that $\mathrm{Co}^{2+}$ ions provide good traps for the excited electrons, which give rise to their potential use in nonlinear optics, optoelectronic devices, solar cells, photodetectors and light-emitting diodes [1-3]. By doping nanocrystals, the impurity states can play a special role in affecting the electronic energy structures and transient probabilities. Indoped nanocrystals the luminescence quantum efficiency is expected to increase as a result of a greater interaction between the electron and the hole of the host semiconductor material with the localized dopant levels [4]. However, effect of Mn doping on the structural phase and size control and optical properties of ZnS nanostructures is still an issue and many groups have focused on this problems [5].

This paper presents some interesting results obtained on the synthesis of $\mathrm{ZnS}: \mathrm{Co}$ nanocrystals by the aqueous solution method with controllable and narrow size distribution. We treat the effect of Co doping on the luminescence properties of $\mathrm{ZnS}$ nanoparticles. Also, we consider the effect of various synthesizing conditions on the narrow size distribution of the $\mathrm{ZnS}$ :Co particles and the luminescence intensity.

\section{Experimental}

Aqueous solutions of zink chloride, cobaltchloride and the capping agent thioglycerol (TG) of high $(0.1 \mathrm{M})$ and low $(0.01 \mathrm{M})$ concentrations are prepared in ethanol. The $\mathrm{pH}$ was adjusted to about 2-10 by adding appropriate amounts of $\mathrm{NaOH}$, before adding TG.Sodium sulfide dissolved in distilled water was added dropwise to the mixture for $5 \mathrm{~min}$. The total reaction time was about $2 \mathrm{~h}$. Nitrogen gas was used for deoxygenating the reaction vessel. The temperature in different experiments ranged 
between $300^{\circ} \mathrm{C}$ and $700 \mathrm{C}$. In high temperature experiments, the synthesis vessel was heated in the bath. The synthesis solution was washed with acetone to get rid of unreacted ions remaining outsidethe clusters and then was centrifuged. Finally, the precipitate was air-dried to get a powder of ZnS:Co nanoparticles. The optical absorption spectra of ZnS:Co nanocrystallites dispersed in ethanol were recovered using a UV-Vis spectrophotometer (Cary 100). The luminescence spectra were recorded on a LF-5 Perkin Elmer spectrometer with the excitation wavelengths of about $370 \mathrm{~nm}$ to excite the ZnS:Co nanocrystals.

\section{Results and Discussion}

Deposited films were kept "as it were" for the other characterization like XRD, AFM and XRF. Thickness of the films was measured by gravity method. Thickness for ZnS, ZnS:Co films was varied from $84-85.26 \mathrm{~nm}$.

Figure 1. shows the XRD patterns of ZnS:Co thin films deposited at fixed $\mathrm{pH}$ value and temperature of $70^{\circ} \mathrm{C}$. The XRD peaks appear at $33.20^{\circ}, 47.32^{\circ}, 56.36^{\circ}$ and $69.4^{\circ}$ in the pure ZnS film corresponding to the diffraction from (200), (220), (311), (400) planes respectively of the cubic phase of $\mathrm{ZnS}$. Results are very much closer to the value of JCPDS card file 05-0566. Same diffraction peaks are also observed in the $\mathrm{ZnS}$ :Co films except in the $0.05 \mathrm{M} \mathrm{CoO}_{3}$ doped film ( $\mathrm{ZnS}: \mathrm{Co}$ ), where the (400) plane is absent. Thus all the deposited films are polycrystalline in nature. From the XRD spectra it is clear that the intensity of (200) plane in all the films is higher than that of the other peaks which indicates that the orientation of the grain growth is along (200) plane. It has also been observed that the intensity of the (200) plane in doped films is higher than that of the und oped film indicating improvement of the crystallinity in the doped films. However the peak positions in the doped films have been found to be slightly shifted from the undoped one. The shift may be due to presence of extra Co atoms impurity existence in doped ZnS film and also the development of residual stress in different positions of the thin films.

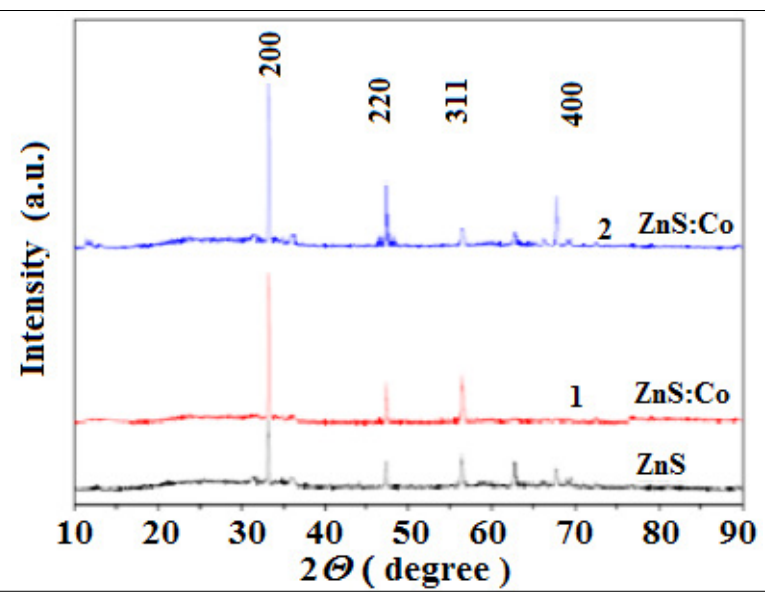

Figure 1. XRD spectra of ZnS:Co undoped nanocrystals, 5\% (1) and $10 \%$ (2) doping nanocrystals $(\mathrm{pH}-4)$

Atomic force microscopy (AFM) is one of the effective ways for the surface analysis of thin films due to its high resolution and powerful analysis software. The $\mathrm{ZnS}$ and $\mathrm{ZnS}$ :Co thin films were morphologically characterized using AFM technique. Figure 2 shows the image of ZnS:Co film in scanned area of $5 \times 5$ and $10 \times 10 \mu \mathrm{m}^{2}$. In both the films the surface is rough and fully covered in the substrate. Clusters of particles are observed over the surface in undoped ZnS film. While formation of unregulated grains of different sizes are observed clearly with pinhole free in ZnS:Co film.

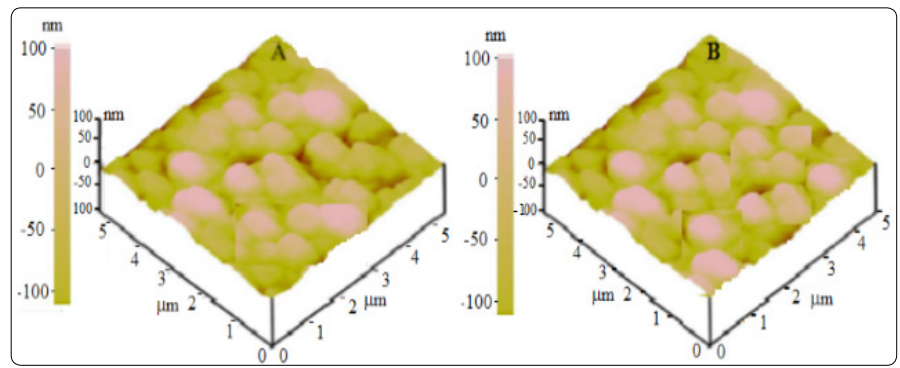

Figure 2. AFM images of (a) ZnS and (b) ZnS:Co films

Optical absorption of Zns and ZnS:Co films have been studied in the wave length range 340-800 nm. Optical absorption studies of semiconducting materials give some information related to band structure. Optical absorption spectra of typical undoped ZnS:Co thin films have been shown in Figure 3. At higher wavelength i.e. at lower energy side, absorption is low towards visible region. However, an increase of absorption is seen in lower wave length (higher energy) side for both types of films. Higher absorbance has been observed in Co doped ZnS films. This may due to the presence of extra states created by Co impurity in the doped films that absorbs more photon energy than that of the undoped film.
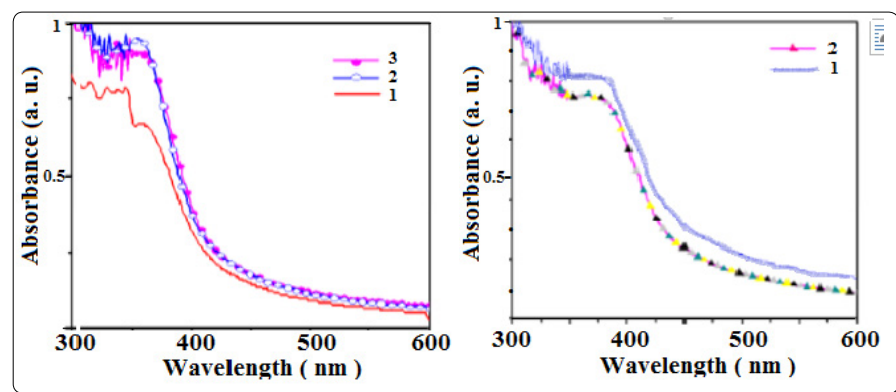

Figure 3. Absorbance spectra of ZnS:Co undoped nanocrystals (1),

$5 \%(2)$ and $10 \%$ (3) doping nanocrystals ( $\mathrm{pH}-4)$ (a) and the luminescence intensity decreases with increasing $\mathrm{pH}(\mathrm{b}): 1-2,2-8$

For a typical nanoparticle sample, luminescence can be generally divided into band edge emission, including excitonic emission, and trap state emission. The luminescence spectra of the ZnS:Co nanocrystals are shown in Figure 4. It was found that the luminescence spectra for all samples could be deconvoluted into two individual components, which corresponded to band edge emission and surface trap state emission [6].

The mechanism of luminescence in $\mathrm{ZnS}$ :Co nanostructure can be explained as following: after excitation, energy will be transferred from the conduction band of ZnS host to the excited charge carriers which are trapped in shallow trap states. These trapped charge carriers are followed by either energy transfer to the excited state of a $\mathrm{Co}^{2+}$ ion, or radiative recombination with a deeply trapped hole at a defect state [7]. 
We explained the synthesis properties of $\mathrm{ZnS}$ :Co samples. In contrast the trap states are located within the semiconductor band gap and hence their emission is usually red-shifted relative to the band edge emission. In addition, the trap state luminescence is often characterized by a large band width reflecting abroad energy distribution of the emitting states. Nonradiative decay pathways associated with unpassivated surface atoms competed with radiative exciton recombination. The characteristic luminescence can consist of relatively sharp emission bands (spectral width typically a few $\mathrm{nm}$ ), but also of broad bands, whose width can exceed $50 \mathrm{~nm}$ in the visible part of the spectrum. Sharp emission bands are characteristic of optical transitions between electronic states with chemical bonding character (almost) the same for ground and excited states that hardly participate in the chemical bonding. These activities in the host material define the luminescence efficiency and can produce a narrowing or broadening of the band width of the spectra. As shown in all figures, we have three peaks that can correspond to band edge, shallow traps, and deep traps. Figure 4 shows the PL spectra and absorption spectra of a series of samples with different doping concentration. The PL spectra (Figure $4 a$ ) of the nanocrystal samples show three peaks, at 444,478 , and $530 \mathrm{~nm}$, which are attributed to the band edge, shallow and deep traps, respectively. We see that undoped nanocrystals have a narrower size distribution than the $5 \%$ and $10 \%$ doping nanocrystals.
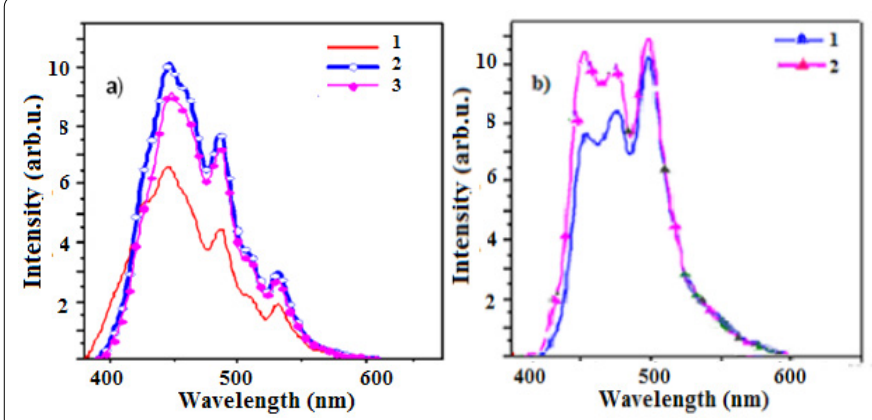

Figure 4. Luminescence spectra of ZnS:Co undoped nanocrystals (1), $5 \%$ (2) and $10 \%$ (3) doping nanocrystals ( $\mathrm{pH}-4$ ) (a) and the luminescence intensity decreases with increasing $\mathrm{pH}(\mathrm{b}): 1-2,2-8$

Therefore they have the highest emission intensity. But we expected doping Co ions to produce better luminescence spectra. The impurity concentrations generally are low in view of the fact that at higher concentration the efficiency of the luminescence process usually decreases (concentration quenching). It may be that, at high concentration, there is the broad size distribution of nanocrystals and broad emission wavelength, and as a result, low luminescence intensity. As we show in figure $4 \mathrm{~b}$, the luminescence intensity decreases with increasing $\mathrm{pH}$. The absorption spectra in $\mathrm{pH} 4$ have sharper excitonic peaks than $\mathrm{pH} 2$ and 10, respectively. Moreover, it was found that $\operatorname{CoS}$ can create an acidic synthesis solution which produces radiative recombination centers in the $\mathrm{ZnS}$ host material. We prepared ZnS:Co nanocrystals at a high precursor concentration with $0.1 \mathrm{M}$.
It means that doping of nanocrystals occurs completely in high precursor concentration. Also, we investigated the optimize conditions for the best luminescence with different excitation wavelengths and doping concentrations there is a narrowing of the excitonic absorption peak at $350 \mathrm{~nm}$. We excited sample $\mathrm{K}$ with three excitation wavelengths. There is the best luminescence for $360 \mathrm{~nm}$ excitation which is near to band edge wavelength. Two other excited emissions related to the sizes of other nanoparticles were distributed in this sample. We find a red-shift of the band edge emission by varying the excited wavelength. But we have abroad peak at $465 \mathrm{~nm}$ in all of them that is related to the incorporation of nonradiative and radiative recombination.

\section{Conclusion}

In conclusion, a simple aqueous solution method has been developed for synthesizing the size-controlled $\mathrm{Co}^{2+}$ doped $\mathrm{ZnS}$ nanocrystals with a relatively narrow size distribution. These results indicate that band edge emission is more affective than traps on the luminescence intensity. They do not have noticeable changes in energy by variation of the doping concentrations. It is observed that the luminescence intensity increases with increasing Co concentration from $1 \%$ to $5 \%$. Luminescence intensity in different excitation wavelength shows portion of different size of $\mathrm{ZnS}: \mathrm{Co}$ nanocrystals on luminescence spectra. We found that by narrowing the size distribution and the amount of doping concentration $\mathrm{ZnS}$ samples with a high luminescence intensity can be prepared.

\section{References}

1. Bhargava RN, Gallagher D, Welker T. Doped nanocrystals of semiconductors - a new class of luminescent materials. J. Lumin. 1994; 60(61): 275-280. doi: 10.1016/0022-2313(94)90146-5

2. Lu H, Chu SYS. The mechanism and characteristics of ZnS-based phosphor powders. J. Cryst. Growth. 2004; 265(3-4): 476-81. doi: 10.1016/j. jcrysgro.2004.02.011

3. Arai T, Senda S, Sato Y, et al. Cu-doped ZnS hollow particle with high activity for hydrogen generation from alkaline sulfide solution under visible light. Chem. Mater.2008; 20(5): 1997-2000. doi: 10.1021/cm071803p

4. Bhattacharya S, Chakravorty D. Electrical and magnetic properties of cold compact iron doped zinc sulfide nanoparticles synthesised by wet chemical method. Chem. Phys. Lett. 2007; 444: 319.

5. Yu J, Liu H, Wang Y, Jia W. Hot luminescence of $\mathrm{Mn} 2+$ in $\mathrm{ZnS}$ nanocrystals. J. Lumin.1998; 79: 191.

6. Huang J, Yang Y, Xue S, Yang B, Liu S, Shen J. Photoluminescence and electro luminescence of $\mathrm{ZnS}$ :Cu nanocrystals in polymeric networks. Appl. Phys. Lett.1997; 70: 2335. doi: 10.1063/1.118866

7. Jian W, Zhuang J, Zhang D, Dai J, Yang W, Bai Y. Synthesis of highly luminescent and photostable ZnS:Ag nanocrystals under microwave irradiation. Mater. Chem. Phys. 2006; 99(2-3): 494-97. doi: 10.1016/j. matchemphys.2005.11.030 